\title{
Perception and Adoption of Mobile Accessibility Features by Older Adults Experiencing Ability Changes
}

\author{
Rachel L. Franz ${ }^{1} \quad$ Jacob O. Wobbrock $^{1} \quad$ Yi Cheng $^{1} \quad$ Leah Findlater $^{2}$ \\ ${ }^{1}$ The Information School, ${ }^{2}$ Department of Human-Centered Design \& Engineering \\ DUB Group \\ University of Washington \\ Seattle, WA, USA 98195 \\ \{franzrac, wobbrock, chengy23, leahkf\}@uw.edu
}

\begin{abstract}
To investigate how older adults perceive ability changes (e.g., sensory, physical, cognitive) and how attitudes toward those changes affect perception and adoption of built-in mobile accessibility features (such as those found on Apple iOS and Google Android smartphones and tablets), we conducted an interview study with 14 older adults and six of their family members. Accessibility features were difficult for participants to find and configure, which were issues compounded by a reluctance to use trial-and-error. At 4-6 weeks after the interview, however, some participants had adopted new accessibility features that we had showed them, suggesting a willingness to adopt once features are made visible. The older adults who did already use accessibility features had experienced a disability earlier in life, suggesting that those experiencing progressive ability changes later in life might not be as aware of accessibility features, or might not have the know-how to adapt technologies to their changing needs. Our findings provide support for creating technologies that can detect older adults' abilities and recommend or enact interface changes to match.
\end{abstract}

\section{Author Keywords}

Older adults; changing abilities; accessibility; mobile devices; smartphones; interviews; ability-based design.

\section{ACM Classification Keywords}

- Human-centered computing Empirical studies in

HCI • Human-centered computing Accessibility

technologies - Social and professional topics $\sim$ Seniors

\section{INTRODUCTION}

Older adults are currently the fastest growing demographic group worldwide, and this group will more than double in the next 30 years [42]. As a result, a substantial portion of the population will experience agerelated ability changes, including sensory (vision,

Permission to make digital or hard copies of all or part of this work for personal or classroom use is granted without fee provided that copies are not made or distributed for profit or commercial advantage and that copies bear this notice and the full citation on the first page. Copyrights for components of this work owned by others than ACM must be honored. Abstracting with credit is permitted. To copy otherwise, or republish, to post on servers or to redistribute to lists, requires prior specific permission and/or a fee. Request permissions from Permissions@acm.org.

ASSETS '19, October 28-30, 2019, Pittsburgh, PA, USA

(C) 2019 Association for Computing Machinery.

ACM ISBN 978-1-4503-6676-2/19/10 ..\$15.00

https://doi.org/10.1145/3308561.3353780 hearing), physical (mobility, dexterity), and cognitive (memory, attention) changes.

In addition to this trend, a large portion of older adults are adopting mobile smart devices. In 2017, a Pew survey estimated that $42 \%$ of older adults in the United States owned a smartphone, up from $22 \%$ in 2013 [2].

Most mobile devices have features that can improve their accessibility by making them easier to see, hear, and manipulate. Many older adults who are experiencing ability changes could benefit from these accessibility features. While mobile devices specifically designed for older adults do exist (e.g., https://www.greatcall.com), adoption of accessibility features on a mainstream smartphone carries less of the stigma that is associated with use of assistive technologies [1,5,12,31].

Despite the prevalence of these two trends (an aging population and increased mobile device adoption), we do not yet know the extent to which mobile device accessibility features fit the needs of older adults experiencing gradual ability changes. Unlike people who experience acute physical or sensory disabilities at a young age, older adults might not be entirely aware of their abilities changing, especially if their abilities change gradually over time. They might not have ever considered themselves as even having a disability of any kind, and therefore might not have considered exploring accessibility options on their smart devices.

To understand how older adults who identify as experiencing ability changes use and perceive accessibility features, we conducted interviews with 14 older mobile device users. The interviews covered attitudes towards perceived ability changes, use and perception of accessibility features, and how attitudes impact use. During the study session, participants were also introduced to a range of accessibility features on their own mobile devices. Given that people perform more impression management as they age (i.e., behaviors stemming from the desire to portray oneself in a positive light) [29], we also interviewed family members of six older adult interviewees to gain a second perspective on the older adults' attitudes towards aging and accessibility. Finally, four to six weeks after the initial interview, we conducted a follow-up interview with older adults to investigate whether they had adopted any accessibility 
features, and how their perceptions towards these features had changed.

We found that older adults in our study experienced challenges remembering how to access and configure accessibility features, a problem that was compounded by a hesitation to use trial-and-error. However, at the follow-up interview, three older adults had adopted mobile accessibility features that we had showed them, indicating that when accessibility features are made visible and understood, they might be of lasting value. Only three of 14 older adults had adopted accessibility features prior to our initial interview. These three older adults had all experienced a disability or ability changes before age 65, suggesting that older adults experiencing progressive ability changes for the first time later in life may not be as aware of accessibility features or have the skills to adapt technologies to their changing needs.

This paper contributes what is, to the best of our knowledge, the first empirical investigation of attitudes toward, and adoption of, smart-device accessibility features by older adults who perceive that they are experiencing progressive ability changes. Unlike most accessible computing research, which focuses on acute disabilities, such as those from birth or injury, our focus is on perceived gradual ability decline, which is universally present in the aging process, and yet receives little attention precisely because of its gradual nature.

\section{RELATED WORK}

Related prior work can be grouped into four areas. We summarize each of these in turn.

\section{Factors Influencing Technology Adoption and Use by Older Adults}

Established models of technology adoption can be used to understand older adults' adoption and use of technology, and, as such, have informed our study design. The Technology Acceptance Model (TAM) developed by Davis [8] explains technology adoption through three main components: "Perceived Ease of Use," "Perceived Usefulness," and "Behavioral Intention." This lattermost component relates to the user's attitude towards learning and intention to use technology, which may be particularly relevant when studying how attitudes about ability changes influence feature adoption.

Other researchers have identified additional factors that apply specifically to older adults. For example, Neves et al. [22] address the limitations of TAM by identifying factors such as social context that influence adoption for older adults who are institutionalized and above 80 years old. Functional value, enjoyment, safety, security, and independence can also influence technology adoption by older adults [7]. Barriers to adoption include poor accessibility, age-related functional decline, and poor ease-of use [15,20,30]. In addition, research has found that individuals have difficulty using mobile accessibility features [3,25]. Despite accessibility being identified as a barrier in previous work, our study is the first to investigate older adults' awareness and perception of accessibility features.

\section{Interface Design for Individuals Experiencing Ability Changes}

Research suggests that ability changes are important to consider when designing interfaces. Piper, Brewer, and Cornejo [27] found that for older adults experiencing vision loss in later life, the changing landscape of accessible technology was a barrier for adoption to ICT's, especially because their vision was also changing. They suggested that the design of ICT's should accommodate daily and long-term fluctuations in vision. In line with this finding, Kane et al. [14] found that participants' ability to use their devices were subject to day-to-day fluctuations due to stress, medication, fatigue, and weather, in addition to longer term changes in abilities. Martin-Hammond et al. [18] explored how adaptive user interfaces should be designed for individuals who have variable pointing performance due to dexterity changes. They found that younger and older adults had diverse preferences when it came to notification characteristics, information, language, and discretion. For example, participants wanted a highly visible notification when the system sensed a change in their pointing performance.

\section{Assistive Device Adoption and Use by Older Adults}

Fear and experience of stigma is a main barrier to older adults' adoption of assistive devices. Some older adults resist using assistive devices such as canes and walkers due to the fear of being perceived as frail and dependent $[1,5,6,12]$. Pino et al. [26] found that older adults with mild cognitive impairment were concerned that if they used socially assistive robots, they would be seen as less deserving of human companionship and assistance. Other studies have found that older adults perceive personal alarm devices negatively because these devices are associated with aged care [33,39]. As a result of older adults' preferences for devices that are not associated with old age and dependence, Lee and Coughlin [16] suggested integrating assistive features into mainstream devices.

\section{Mobile Accessibility for Older Adults}

Examining touchscreen interactions, researchers have found that older adults make comparable errors to younger adults $[9,34]$. Yet, older adults tend to perform touchscreen gestures and tasks more slowly than younger adults [9,34]. Findlater et al. [9] found that older adults had a greater gain in performance when using touchscreen devices compared to younger adults, suggesting that touchscreen input might be more accessible for older adults compared to desktop input. Piper et al. [28] also found that older adults could perform tasks on large touchscreen surfaces; however, one challenge was recalling touchscreen gestures. To improve the accessibility of touchscreens for older adults with finger tremor, Wacharamanotham et al. [38] introduced Swabbing, an interaction technique in which the user taps, slides his or her finger towards the target, then lifts off. A controlled study showed that Swabbing lessened finger tremor, and improved error rates and user satisfaction. 


\begin{tabular}{|c|c|c|c|c|c|c|c|c|}
\hline \multicolumn{2}{|c|}{ Identifier Age } & \multirow{2}{*}{$\begin{array}{l}\text { Gender } \\
\mathrm{F}\end{array}$} & \multirow{2}{*}{$\begin{array}{l}\text { Previously }(\mathrm{P}) \text { or } \\
\text { Currently }(\mathrm{C}) \\
\text { Employed } \\
\mathrm{P}\end{array}$} & \multirow{2}{*}{$\begin{array}{l}\text { Occupation } \\
\text { Clinical Psychologist }\end{array}$} & \multirow{2}{*}{$\begin{array}{l}\text { Highest Level } \\
\text { of Education } \\
\text { Ph.D. }\end{array}$} & \multirow{2}{*}{$\begin{array}{l}\text { Ability } \\
\text { Changes } \\
\text { VHMDC }\end{array}$} & \multirow{2}{*}{$\begin{array}{l}\text { Disabilities Developed } \\
\text { Before Age } 65 \\
\text {-- }\end{array}$} & \multirow{2}{*}{$\begin{array}{l}\text { Relationship } \\
\text { to Older } \\
\text { Adult } \\
--\end{array}$} \\
\hline $\mathrm{P} 1$ & 70 & & & & & & & \\
\hline $\mathrm{P} 2$ & 93 & $\mathrm{M}$ & $\mathrm{P}$ & Librarian & Master's & HMDC & -- & -- \\
\hline P3 & 65 & $\mathrm{~F}$ & $\mathrm{P}$ & Rehabilitation Counselor & Some College & VMD & Cerebral Palsy (since birth) & -- \\
\hline P4 & 79 & $\mathrm{~F}$ & $\mathrm{P}$ & Psychologist & Ph.D. & VC & -- & -- \\
\hline P5 & 90 & $\mathrm{M}$ & $\mathrm{P}$ & Consultant & Ph.D. & VHC & -- & -- \\
\hline P6 & 86 & $\mathrm{~F}$ & $\mathrm{P}$ & Teacher & Master's & VM & -- & -- \\
\hline P7 & 65 & $\mathrm{~F}$ & $\mathrm{P}$ & Switchboard Operator & High school & -- & Blindness (since birth) & -- \\
\hline P8 & 78 & $\mathrm{~F}$ & $\mathrm{C}$ & Adoption Specialist & Masters & VHMDC & -- & -- \\
\hline P9 & 75 & $\mathrm{~F}$ & $\mathrm{P}$ & Shop Clerk & High school & VHMC & -- & -- \\
\hline P10 & 67 & $\mathrm{~F}$ & $\mathrm{P}$ & Corporate Communications & Associate's & VHMDC & Paraplegia (age 45) & -- \\
\hline P11 & 79 & M & $\mathrm{P}$ & Developer & Master's & VHC & -- & -- \\
\hline $\mathrm{P} 12$ & 76 & $\mathrm{~F}$ & C & Executive Director & Bachelor's & VHC & -- & -- \\
\hline $\mathrm{P} 13$ & 81 & $\mathrm{~F}$ & $\mathrm{P}$ & Registered Nurse & Master's & VHC & -- & -- \\
\hline $\mathrm{P} 14$ & 75 & $\mathrm{~F}$ & $\mathrm{P}$ & Hospital Administration & Master's & $\mathrm{VHC}$ & Blindness in one eye (age 34) & -- \\
\hline$\overline{\mathrm{F} 1}$ & 65 & $\mathrm{~F}$ & $\mathrm{P}$ & Technical Communications & Bachelor's & 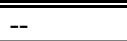 & -- & Sister \\
\hline F3 & 27 & $\mathrm{~F}$ & C & Occupational Therapist & Master's & -- & -- & Daughter \\
\hline F5 & 61 & $\mathrm{M}$ & C & Senior Center Manager & Master's & -- & -- & Son \\
\hline F10 & 67 & $\mathrm{M}$ & $\mathrm{P}$ & Engineer & Some College & -- & -- & Husband \\
\hline F12 & 79 & $\mathrm{M}$ & $\mathrm{P}$ & Lobbyist & Master's & -- & -- & Husband \\
\hline F13 & 54 & $\mathrm{~F}$ & C & Academic Coach & Master's & -- & -- & Daughter \\
\hline
\end{tabular}

Table 1. Older adult and family member demographic information from our study. Older adult identifiers begin with " $P$ " and family member identifiers begin with " $F$ ". Family member numbers correspond with their older adult's number (e.g., F5 is the family member of P5). Ability Changes are abbreviated thusly: vision=V, hearing=H, mobility of arms, hands, or fingers $=\mathbf{M}$, dexterity $=\mathrm{D}$, cognitive $=\mathrm{C}$.

Beyond basic input, researchers have identified some tasks on mobile devices that are particularly challenging for older adults [21]. To address the challenge of configuration, for example, Olwal et al. [23] developed OldGen, a system that enables caregivers to customize the user interface layout and buttons on generic mobile phone platforms. To improve the accessibility of touchscreen keyboards for older adults, Toshiyuki et al. [13] proposed Typing Tutor, a system that detects common mistakes and offers typing instructions based on the individual's mistakes. Older adults' typing proficiency increased with Typing Tutor, especially during the initial stages of learning.

These studies assess mobile device accessibility or propose new systems that improve accessibility for older adults. Yet, how older adults perceive and adopt built-in accessibility features is not yet understood. That is the purpose of our study.

\section{STUDY METHOD}

Through an interview study with older adults and family members, we investigated older adults' perceptions toward and adoption of mobile accessibility features. We also investigated how older adults' attitudes towards perceived ability changes impact their receptiveness to mobile accessibility features. Our goal is to better understand older adults' perceptions and behaviors, thereby revealing opportunities to tailor technology designs to better meet older adults' needs. For example, we can tailor presentation and instructional materials to older adults, recognizing their accessibility needs and self-perceptions as distinct from other people with disabilities that did not arise gradually as a result of aging.
We investigate self-reported awareness of ability changes, as we are concerned with participants' self-perceptions, attitudes, and willingness to adopt accessibility features, even if ability changes are not objectively verifiable through an interview study utilizing self-reports.

\section{Participants}

The inclusion criteria were that participants (1) were $65+,(2)$ owned or used a smart device, and (3) perceived that they were experiencing a change in abilities in the past five years and/or experiencing an ongoing disability. Fourteen older adults aged 65 to $93(M=77.1, S D=19.7)$ participated in our study. As shown in Table 1, all perceived that they were experiencing ability changes within the past five years and/or were experiencing an ongoing disability (i.e., blindness). Two older adults (P3 and P7) had lived with a disability their entire lives while two developed a disability before turning 65 (P10 and P14). P12 worked in an aging-related career for much of her life, so in addition to her personal experiences, she had professional knowledge of age-related changes faced by older adults. All older adults owned and used a mobile device (five used Android phones, eight used iPhones, and one used a Kindle), which was a requirement for participation.

Because older adults might perform impression management [29] when discussing their abilities, which in turn could affect the accuracy of their self-report data, we also recruited a family member for corroboration when possible. This family member (e.g., spouse, sibling, adult child) was interviewed independently to provide a second perspective on the older adult's experiences. We were able to recruit a family member for six of 14 of the older adult participants. Family members were $27-79$ years old $(M=58.8, S D=17.6)$ (Table 1). 


\begin{tabular}{|c|c|c|c|}
\hline $\begin{array}{l}\text { Related } \\
\text { ability }\end{array}$ & $\begin{array}{l}\text { Feature (Available on } \\
\text { Android }(A) \text {, iOS (I), or } \\
\text { Kindle }(K))\end{array}$ & Feature description & $\begin{array}{l}\text { Participants who already use }(\mathrm{U}) \text {, } \\
\text { are aware of but do not use }(\mathrm{A}) \text {, } \\
\text { or were introduced to the feature } \\
\text { during the interview }(\mathrm{I})\end{array}$ \\
\hline \multirow[t]{9}{*}{ Vision } & Talkback (A), VoiceOver (I) & Provides spoken feedback about information on the screen & P5I, P7U, P12A \\
\hline & $\begin{array}{l}\text { Select to speak (A), speak } \\
\text { selection (I) }\end{array}$ & Reads selected text aloud & P4I, P6I, P8I, P11I \\
\hline & Display size $(\mathrm{A})$ & Increases/decreases parts of the display (e.g., icons) & P3I, P5U*, P11I \\
\hline & $\begin{array}{l}\text { Font size }(\mathrm{AK}) \text {, larger text } \\
(\mathrm{I}), \text { bold text }(\mathrm{I})\end{array}$ & Increases/decreases the font size or weight & $\begin{array}{l}\text { P2I, P3A, P5U*, P6U, P8U, P10I, } \\
\text { P11I, P12A, P13U, P14U }\end{array}$ \\
\hline & $\begin{array}{l}\text { Magnification gestures }(\mathrm{A}) \text {, } \\
\text { zoom magnification }(\mathrm{I})\end{array}$ & Magnifies the screen (works when pinch to zoom does not) & P3A, P10I, P11I, P12I, P13I, P14I \\
\hline & Contrast options (A) & Increases/decreases the contrast of the display & P3A, P11I \\
\hline & $\begin{array}{l}\text { Color options }(\mathrm{A}) \text {, display } \\
\text { accommodations }(\mathrm{I})\end{array}$ & Inverts or corrects the display colors for colorblindness & $\begin{array}{l}\text { P1U, P3I, P6I, P7U, P10I, P12I, } \\
\text { P13I }\end{array}$ \\
\hline & Magnifier (I) & Opens the camera to act as a magnifying glass. & P2I, P8I, P10I, P12I, P13I \\
\hline & Speak passwords (A) & Speaks out password & $\mathrm{P} 14 \mathrm{I}$ \\
\hline \multirow[t]{3}{*}{ Hearing } & Captions (AI) & Layers text over video contents & P11I \\
\hline & Pair hearing aid $(\mathrm{I})$ & Pairs hearing aid with device to control the hearing aid & $\mathrm{P} 12 \mathrm{U}, \mathrm{P} 13 \mathrm{I}$ \\
\hline & Flash notification (I) & Flash blinks when receiving calls or messages & P13U* \\
\hline \multirow[t]{5}{*}{ Mobility } & Speech to text $(\mathrm{Al})$ & Converts user's speech into text & $\begin{array}{l}\text { P1U, P2A, P3U, P4A, P5A, P6I, } \\
\text { P7U, P8I, P9I, P10I, P11I, P12U, } \\
\text { P13A, P14A }\end{array}$ \\
\hline & Voice record messages $(\mathrm{Al})$ & Records a short audio clip and sends in a text message chat & P4U, P8I, P9I \\
\hline & Assistive Touch (I) & $\begin{array}{l}\text { Allows users to control the device without using physical } \\
\text { buttons or gestures }\end{array}$ & P8I, P10I, P12I, P13I, P14U \\
\hline & Touch and hold delay (A) & Adjusts the timing for a touch and hold gesture & P9l \\
\hline & Power button ends call (A) & Pressing the power button will end the call & P14l \\
\hline
\end{tabular}

Table 2. Accessibility and mainstream features that improve accessibility found on Google Android, Apple iOS, and Amazon Kindle. *The participant was using the feature without knowing it had been configured by a family member.

\section{Theory}

To investigate adoption and use of mobile accessibility features, we integrated components from two established models used to explain and predict technology adoption and continuous use: The Technology Acceptance Model (TAM) [8] and the Unified Theory of Acceptance and Use of Technology (UTAUT) [36]. In addition to these established frameworks, we reviewed factors reported by Neves et al. [22], which are specific to older adults.

Old age is a dynamic phase in life. Commonly, old age is portrayed as a binary state in HCI research (e.g., one is either $65+$ or not). In addition, older adults are often characterized as impaired and dependent [37]. To avoid the pitfalls of conceptualizing abilities as static and older adults as disabled, we applied the life course perspective [10] to gain insight into how previous life experiences and circumstances impact current ones. Additionally, this framing allows for the possibility of some abilities actually improving with age.

Finally, we adopted an Ability-Based Design perspective $[40,41]$, focusing on what older adults can do and not what they cannot do. Thus, our interviews were not only concerned with the challenges and limitations older adults encounter, but their abilities, capabilities, and interests.

\section{Procedure}

We conducted semi-structured interviews with both older adults and family members. The interview questions were identical for both groups, except that we asked the family member questions about the older adult instead of about themselves. Older adult and family member interviews were conducted separately. The interviews lasted an average of 67 minutes $(S D=17)$ for older adults and 41 minutes $(S D=12)$ for family members. Participants were asked to bring their own mobile devices to the interviews. The interview was structured in five parts: we asked about older adults': (1) perceptions toward aging and changing abilities, (2) adoption and use of mobile devices, (3) how ability changes impacted their mobile device use, (4) how they configured their devices to make them more accessible, and (5) we walked through accessibility features that could be useful for them based on what they had said about their abilities (see supplementary material for the interview script). During the walkthrough, we asked what the participant thought of the feature and, with the older adults, whether we should leave the feature enabled on their phone. We conducted the walkthrough on the participant's device unless they did not have it (two participants), in which case we used a device with the same operating system as the participant's device. Table 2 shows which features had already been adopted by participants and which features were demonstrated in the walk-through. We made assessments of which features would be potentially useful for the participant during the session.

The interview was informed by UTAUT and TAM constructs, specifically, social support and influence (part 2), facilitating conditions (part 2 and 3), perceived ease of use (part 3, 4, and 5), perceived usefulness (part 3, 4, and 5), and behavioral intention (part 5). All interviews were audio recorded. 
Four to six weeks after the older adults' first interviews, we followed-up via phone interviews to understand participants' perceptions and adoption or continued use of accessibility features. These follow-up interviews lasted about 10 to 20 minutes. The researcher took notes of the follow-up call. Only one participant, P4, declined to do a follow-up interview due to a family emergency.

\section{Data Analysis}

We transcribed the audio-recorded interviews and conducted an inductive and deductive thematic analysis on the transcripts [24]. Prior to the thematic analysis, we identified five deductive themes from TAM [8], UTAUT [36], and Neves et al. [22] relating to factors that contribute to adoption and continued use: attitudinal factors, digital literacy, perceived ease of use, perceived usefulness, and social support and influence. The first and third authors paraphrased participant responses in the transcripts to make the responses stand alone without requiring context from the rest of the interview, in a format similar to interpretation session notes from Beyer and Holtzblatt's Contextual Design method [4]. The first author then independently developed a codebook based on the paraphrased utterances from three transcripts. The third and fourth authors conducted a quality check on the codebook to test for convergence. After refining the codebook, the first author independently coded the remaining interviews. The third author performed inter-rater agreement on four randomly selected initial interview transcripts, finding that Cohen's kappa was 0.82 . Notes from the follow-up calls were coded using the codebook from the initial interviews.

\section{RESULTS}

We organize our findings into three main sections: (1) ability changes and how these changes affect device use; (2) awareness, use, and perceptions of accessibility features; and (3) longer-term adoption of accessibility features. Although we found codes that matched our deductive themes (i.e., Attitudinal Factors, Digital Literacy, Perceived Ease of Use, etc.) we chose not to organize the paper around these factors because these are already known in the literature. Instead, we focused our organization to align with our research question, namely, how do older adults who identify as experiencing ability changes use and perceive accessibility features?

When "participants" are referred to in general, these are members of our primary participant group of older adults. Family member participants are always referred to explicitly as being family members.

\section{Older Adults' Ability Changes and How Ability Changes} Affect Device Use

We identified main themes including: perceptions of ability changes, how ability changes affect device use and willingness to explore, and how technology could support older adults' future ability changes.

\section{Perceptions of Ability Changes}

All older adults had mixed perceptions of aging and most family members agreed with older adults' perceptions. To infer the participants' dominant attitudes toward their ability changes, we inductively categorized and counted perceptions toward ability changes into three main categories: positive, negative, and relative. We labelled older adults' dominant attitudes based on the category that contained the most utterances. Many had a positive attitude towards their abilities changing (P4, P5, P6, P8, P12, P13). Participants who had predominantly positive attitudes had accepted that their abilities were changing, were able to adapt to the changes, and recognized that they could still do the things they wanted to do (e.g., attend a reunion). Some participants had a negative attitude (P1, P3, P10, P11, P14), These participants expressed their frustration with not being able to do things as well as before and were fearful of becoming dependent. A few participants viewed their abilities changing in relation to other people and expectations for themselves, sometimes comparing positively or negatively (P2, P9, P12). These participants normalized their abilities relative to what they believed to be "average" for their age or compared their abilities to younger or older people.

Some of the family members agreed with older adults' attitudes, while F5, F12, and F13 disagreed. Although P5, P12, and P13 had predominantly positive attitudes towards aging, their family members believed that their older adults had mixed attitudes. For example, family members noticed that their older adult was frustrated (F5), anxious (F13), or irritated (F12) with their ability changes.

\section{How Ability Changes Affect Device Use}

Although all older adults owned mobile devices, had them for an average of 5 years $(S D=4.4)$, and used them for a variety of purposes (e.g., communication, games, reading etc.), they reported challenges using devices due to cognitive, vision, hearing, mobility, and dexterity changes.

Cognitive Changes: Cognitive changes affected participants' use in a variety of ways, such as increased difficulty learning due to a concussion ( $\mathrm{P} 4)$, increased resistance to learning (P5), and increased difficulty troubleshooting (P3).

To compensate for cognitive changes, some older adults started to rely more on calendar, notes, and reminder apps (P4, P10, P11, P13). However, P4 and P13 did not think these apps helped them: P4 would accidentally enter the wrong information; P13 sometimes forgot appointments even after being notified and would accidentally disable notifications. P5 and P11 did not save any information on their phones and instead relied on physical notebooks because P5 found using physical notebooks easier, while P11 did not trust that his phone would save his notes.

Vision Changes: Vision changes affected participants' ability to read on their devices. P2, P3, P6 and P13 complained that the font size was too small. P6 and P14 said that it was more difficult reading on the phone when the contrast was low. To compensate for changes in vision, P3, P8 and P13 reported that they started to put on glasses before using their devices. P14 was blind in one eye and experienced haziness in the other eye, and as a result had to hold devices six inches from 
her face to see through the haziness in her vision, which was something she did not have to do before her vision started changing. P2 bought a large phone to read more easily on it. $\mathrm{P} 11, \mathrm{P} 12$ and $\mathrm{P} 13$ reported that they preferred reading on their tablets because of the bigger screen.

P6 had developed macular degeneration, which inhibited her ability to see fonts with curvilinear features. She abandoned her Apple iPad as a result of not being able to see the font. P3 also tried to change the font face on her device due to difficulty reading but was unable to. She explained:

"I do a lot better seeing with Arial. [...] I believe it's because it doesn't have many curlicues on things and it's really basic lettering."

Hearing Changes: Most participants except for P10 and P12 did not think that their hearing changes affected their mobile device use. P10 started using the speaker phone as a result of hearing loss while P12 used a Bluetooth headset rather than the phone's ear speaker.

Dexterity and Mobility Changes: Some participants' ability to type was affected by dexterity changes. For P1, P8, and $\mathrm{P} 13$, the numbness and stiffness in their fingers made it difficult to type. P3 avoided typing on her phone and P1 and P3 started using dictation as result of dexterity changes.

P2 and P10 reported accidentally tapping on things. P8 used her middle finger to tap instead of her index finger because it "felt less clumsy." P1, P2, P3, P10, and P12 said that they frequently dropped their devices and P10 said that "the floor becomes a magnet" when using a wheelchair.

P1, P6, P8, and P10 felt that it was difficult to hold the device while using it. P6 bought a lighter Amazon Kindle and started using pillows to support it while reading. P1 used the speakerphone as a result of pain from holding the phone to her ear due to arthritis in her wrist. P9, who had to wear wrist braces due to osteoarthritis, felt that her hand mobility negatively affected her ability to learn how to use a smartphone.

How Ability Changes Affect Willingness to Explore and Experiment with Technology

Most of the older adults did not explore or use trial-and-error with their devices, except for P3 and P7. P3 suggested that older adults' resistance towards exploration was due to older adults' changes in abilities. P3 explained:

"There is something that slows down your ability to work back and forth between things and things you grew up with that were perfectly natural when your cognition is at its peak."

P5, P9, P13 mentioned that they would ask for social support when trying something new on their devices. Demonstrating this behavior, P9's motivation to participate in the study was at least partly due to the opportunity to learn about her phone with the support of the researcher.

A couple of family members' statements agreed with those of older adults that older adults were hesitant to explore devices and settings: F5 and F10 believed unwillingness to explore was a result of older adults' ability changes. For example, F10 discussed P10 thusly:

Researcher [R]: And then her [P10's] use of her smartphone... Has that changed as a result of her change in dexterity?

F10: Just where she's not willing to try much. She'll just go through the minimal things.

$R$ : And you think that's because of her dexterity?

F10: Yeah, well, dexterity and memory both [laughs].

F5 also reflected this theme, stating that he believed his father (P5) was less willing to explore new things on his phone due to cognitive change:

"I do think that because he gets confused, he is less likely to experiment, or, um, use the device in ways that would make his life easier. [...] And I think that given where he was 10 years ago, if someone would have suggested that to him, like you suggested, that you could [use] voice text messaging today, he would be more likely to adopt it. [...] And that would be helpful, but, um, setting it up is confusing and so he elects not to explore things which he would have."

P3 and P7, who both had a disability early in life, believed that experiencing a severe disability at a young age made them more willing to use and fix technology independently in older age. For example, P7, who is blind, said that she had to learn to fix problems on her device independently because most salespeople did not know how add-on assistive technology programs such as screen readers worked:

"Along with the technology comes the issue of if something doesn't work, a blind person has to figure it out, because how many people know about my speech program? Not many. So, I have to figure out my own computer issues because all these places like Best Buy, and all these computer places, they don't know, they've never had to deal with it. They just say, 'gee, I don't know why it doesn't work.' So, I think I have a more-it's kind of like being your own mechanic. You have to kind of know how things work because you're gonna be the one fixing them."

P3, who has cerebral palsy and uses a wheelchair, felt that her ability to figure out technology improved as she got older because she had to practice patience to overcome challenges related to her motor impairment from a young age:

"I am more experienced. And I am less impulsive and being quick is less important to me than it was when I was younger. And so, I can learn, I can-there is a lot of new skills that are required with technology [...] It's harder but it's a little bit more possible than when I was younger. I would say, 'Oh, that's too hard. I got too many other things to do. I am not gonna worry about that. I am not going to do it;' and now it's kind like, 'Maybe I can do that. Of course I can do that. I just have to; there's 
gotta be a way to do that.' [...] But there is no denying at the end of it that I have less hand function. I just have a better brain."

F3, who worked with special needs students as an occupational therapist, suggested that willingness to explore new technology might be related to the onset of an impairment in someone's life in addition to when that person was introduced to technology.

"Even the younger students with disabilities that I work with that have been exposed to technology will tend to poke around the technology until they find what they want. They're not particularly concerned about making a mistake on it, just a level of comfort and familiarity with it that comes with that."

How Technology Could Support Future Ability Changes Some older adults (P3, P7, P8, P10, P12, and P13) and family members (F1, F3, and F13) mentioned their belief that technology could compensate for age-related ability declines, and that older adults would increasingly become dependent upon technology to maintain independence in their daily lives.

P3, P7, and P10 felt that having a disability early on gave them a deeper appreciation of technology because they had a greater dependence on it over the course of their lives. For example, P3 felt she had to keep up with technology changes in older age as a result of her disability:

$R$ : How do you think your disability has affected your use of technology in older age?

P3: Oh, I know for an absolute fact that it made me want "more, faster, bigger, greater [technology]," because I've had to adapt to things that I can't. I don't have the luxury of being able to do it the way I always done it. "Cause I wasn't able to do it that way to begin with."

For P10, being an older disabled woman made her feel vulnerable, but technology gave her a sense of security as she aged:

"The older you get, the more, for women anyway, you feel more vulnerable, and if you're a disabled woman you feel even more vulnerable. So, having a smartphone with you gives you a little peace of mind."

Although many older adults in the study perceived technology as being critical to their future, this was not the case for all technologies, especially technologies that were perceived as stigmatizing and patronizing. P8 and P14 mentioned that they were reluctant to use assistive technologies such as specialized technology for seniors (e.g., Jitterbug) because they felt it was "dumbed down," that it limited what they could do, that it did not grow with them, and that being able to use mainstream devices promoted a sense of social inclusion.
Awareness, Use, and Perceptions of Accessibility Features and Related Settings

In this section, we discuss how older adults configured their devices to make them more accessible. We also discuss participants' perceptions of the features we demonstrated.

Accessibility Feature Awareness and Use

Most older adult participants knew the meaning of the word "accessibility." Of this group, half associated accessibility with disability unprompted. For example, P14 explained:

$R$ : What does accessibility mean to you?

P14: Well, I hope it's something I won't need. [laughter]

$R$ : Why is that?

P14: [laughs] Because it means my body is falling apart. [...] A friend of mine [...] lost her vision when she was in her 30s from diabetes. [...] She went from being somebody who could see how your computer screen looks and stuff, to being completely blind but still using her computer. There was a lot of verbal feedback, telling her what to do and stuff. I thought, "Oh, my God. What if I have to do that?" It was just horrible to think about. I would rather not think about accessibility; it's not a pleasant idea.

R: Why do you not like thinking about it?

P14: Well, I think I would rather be in denial about potential future losses of abilities. I want everything to stay just the way it is now or not get any worse. I'm not going to think about what might be in the future and that includes accessibility.

P1 observed that the fact that accessibility features were labelled as such was problematic because people of all abilities could benefit from accessibility features. For example, here is an exchange about the screen rotation feature:

P1: Once my brother-in-law found the other [setting] under "accessibility," which seems a strange place to put it. I am thinking maybe the volume and speaker can be adjusted in that same category.

R: Why do you feel it's strange that it would be under "accessibility?"

P1: It doesn't seem to me to be something that only people with accessibility issues will need to use. It seems to me a very straightforward thing.

F3 had a similar sentiment towards the "accessibility" label:

'In that case, I don't know that 'accessibility' is the best name for the category, but it is more than just people with disabilities or impaired abilities accessing it and using it for things. I know plenty of people who turn the text size up a little bit just because a lot of us have bad vision even if we're not old or whatever."

F3 went on to say that the reverse is also true, and some mainstream features such as dictation can be perceived as 
accessibility features because they make the device easier to use for people with motor impairments.

Even though most participants knew the meaning of "accessibility," only P3, P7, and P14 were aware of and used accessibility features on their mobile devices. These participants had experienced a disability in early life, and P3 and $\mathrm{P} 7$ depended daily on mobile accessibility features (e.g., screen reader, screen magnifier). In contrast, P14 was aware of accessibility settings because she had used Apple iOS AssistiveTouch as a work-around when the physical home button on her iPod Touch stopped working.

All family members but F12 knew the meaning of the word "accessibility." F3, F5, F10 and F13 were aware that accessibility settings existed and F3, F5, and F13 had configured accessibility settings for their older adult, so P5 and P13 were using accessibility features without knowing they had already been configured-P5: font and display size; P13: flash notification. Highlighting a challenge with accessibility features, F10 did not think accessibility settings were usable, so he did not show his wife or activate them for her:

R: And why didn't you set [accessibility settings] on her phone? Was there a reason why you didn't mention them to her?

F10: I just thought it would be-she wouldn't do it and... R: Hmm. Why?

F10: Because of her memory and dexterity. So, she wouldn't remember it next time or she'd turn it on, forget to turn it off. That kind of thing.

\section{Related Settings Awareness and Use}

Older adults were aware of and used a range of settings that enhanced the accessibility of their devices but were not labelled as accessibility settings. Such features included font size, brightness, dictation, voice assistants (e.g., Apple Siri, Google Assistant), volume, flashlight, and speaker phone. Table 2 offers a detailed breakdown of awareness and use of accessibility and related settings.

Despite many older adults being unaware of display settings on their mobile devices, some participants were aware of display settings on their personal computers. P3, P11, and P12 used these settings to adjust the brightness and font size and change the typeface. When asked why she had not adjusted the display settings on her smartphone, P12 said:

"Probably because I was ignorant of what all they might do. It didn't occur to me, for example, when I was having a little trouble seeing things on my phone that I could change something."

P3, who had changed the display settings on both computer and phone, said, "It's easier to make changes to my desktop to do that than to do it to the mobile devices."

Impressions of Accessibility Features and Related Settings

The researcher showed participants an average of 3.5 features that would potentially be useful to the older adult based on their reported ability changes (see Table 2). All participants except P1 liked at least one accessibility feature that the researcher demonstrated, including Apple iOS AssistiveTouch (P10, P13, P14), font size (P5, P6, P10, P11, $\mathrm{P} 12, \mathrm{P} 14)$, iOS magnifier (P2, P10, P11, P12, P13, P14), speak selection (P3, P4, P6, P8, and P11), speech to text (P5, P6, P8, P9, P10, P11) and zoom (P10, P11, P13).

$\mathrm{P} 8, \mathrm{P} 10, \mathrm{P} 12$, and $\mathrm{P} 14$ thought it was important to be aware of some features for future ability changes. For P1, we demonstrated ways in which she could enhance the auditory accessibility of her device by increasing the speakerphone volume or pairing her device to an external speaker. However, none of these options addressed her accessibility needs because the volume was still too low on the speaker phone and she did not want to bother with an external speaker.

Despite liking several of the accessibility and other features, participants perceived some challenges that would hinder the use of these features. Challenges included: accessibility features being hard to find, complex to configure, and introducing potential usability issues. P12 felt that setting up and using accessibility features was too complicated:

"I don't know, I would say for the people who need accessibility features the most, it's a little overly complicated."

Another related challenge was remembering how to activate a feature. For example, P2 and F6 thought the magnification feature was useful, "but what I have to do is remember how to use it" (P2). P5 and P9 were also concerned about not being able to remember the steps to use the dictation feature: "Yeah, that might make it a little easier for my fingers. Except if I could remember how to do it [laughs]" (P9). F10 had a similar concern about the dictation feature for his wife:

"When she wants to use it, she has to enable it, usually, and then she'll-I'm sure if she did that, she'll forget to turn it off and she'll be talking to herself and that will go into that thing [...] and so it just doesn't make it real easy."

In terms of Android TalkBack, F5 did not think his father, who had low vision, would be able to remember what items were on his screen after they had been read aloud:

F5: I don't know if that would be particularly helpful to him. [...] Because it's - it would be too much information to figure out. [...] By the time it got [through], he wouldn't remember which one was which one. It went too fast.

Another concern was controlling features. Five participants were concerned about controlling the dictation output. P3 and P12 were concerned about controlling the zoom feature, which is a magnifying lens that appears over the screen in the Apple iOS accessibility features. This feature requires the individual to use multiple fingers and taps to maneuver the zoom lens over the screen: 
"I can increase the font size a little bit. But if you get it, then it's way bigger than the space that it's in; then you're sliding over this way and you're sliding over that way. It's easy to get stuck. I don't know where I am, here or... and I am working so hard doing that, I drop the phone", (P3).

P3 had a motor impairment that made operating the zoom feature especially difficult because it required multi-touch. F3 had further insight based on observing her mother's use:

"I think the zoom and magnify features are kind of clunky and require advanced understanding of how to use them. [It] would be better if it wasn't five clicks in to turn it on and off. Nothing to do with the functionality of the feature, just the ease of access of it" (F3).

The usability of the font size feature was also a challenge for participants because increasing the font size reduced reading speed (P3, P6, P11), as was also found in prior work [35]. Additionally, the font was not increased consistently across all the text.

F3 summarized the usability issues with accessibility features thusly:

"I think [accessibility features] work well once they're set up. [...] [Older adults] don't often, A, know what's available, B, know how to access it, or $C$, know how to access the right ones for them or in the most effective way. They don't know if you turn the font size all the way up that you're going to see [only] two letters on your screen."

\section{Longer-Term Adoption of Accessibility Features}

We followed up with participants four to six weeks after the interview. Five older adult participants continued to use features demonstrated in the interview with varying success (P3, P11, P12, P13, P14). The features these participants liked included speak selection (P11), invert colors (P3), black and white contrast (P3), home button ends call (P14), and Assistive Touch (P14).

P3, P12, P13, and P14 had reduced the font size because they felt the larger text size that had been set during the session hindered their ability to read by slowing them down. P14 felt that it was easier for her to see the text by holding the phone close to her face rather than by increasing the font size, because the "edges were still blurry." P7 was still using Apple VoiceOver.

Many older adult participants (P1, P2, P5, P6, P8, P9, P10) had not used or explored the accessibility and related features after the in-person interview. The most common reason for not using or exploring these features was either because the older adult had forgotten the features existed, or had forgotten how to activate them (P1, P2, P5, P8, P10). The second most common reason was a lack of social support to "train" (P5), "coach" (P8), or "reinforce" (P10) how to use the features. P9 had suffered a loss of mobility in her arm due to a fall since the in-person interview and had stopped using her phone completely. She mentioned wanting to take a class to learn how to use her phone once she recovered.

\section{DISCUSSION}

We discuss how our results indicate that older adults who are experiencing gradual ability changes in later life might have a different understanding of their abilities relative to "disability" compared to people with lifelong disabilities.

\section{How Older Adults Experiencing Ability Changes View Themselves in relation to "Disability"}

Although most participants knew the meaning of "accessibility" and had some forms of ability decline, only a few participants actually used accessibility features. P14 negatively associated accessibility features with disability, saying that she hopes she does not ever need to use them. P1 did not think accessibility features should be labelled as such because they could be useful for people without disabilities. The reason that so few participants used accessibility features seems to be related to how participants perceive their own changing abilities in relation to "disability." They might not see themselves as disabled and might perceive accessibility features as something that people with disabilities use. Two of the three participants who were aware of and used accessibility features prior to the in-person interview had lived with a disability since birth (P3 and P7), which further suggests that how people perceive their own abilities in relation to the concept of disability might impact their adoption of accessibility features. This finding highlights a gap in how accessibility features are positioned as meant for "people with disabilities" and how older adults experiencing progressive ability changes might not view themselves as warranting accommodations labeled as such. Designers can rethink using the term "accessibility" and organize accessibility features in a way that suggests their usefulness regardless of the user's disability status.

\section{Accessibility Features are Difficult to Discover, Locate, and Use}

Accessibility features on mobile devices tend to be difficult to discover, requiring navigation through several layers of menus, which was also reported in previous work, e.g., $[3,25]$. This discoverability challenge is highlighted by our findings: only three participants were even aware of mobile accessibility features. Older adult and family participants were also concerned with the older adult being able to remember the steps to access the accessibility features under the settings menu or remembering how to activate and control the features. These are critical usability challenges. If the number of steps to turn on and off an accessibility feature and to find and re-find it are a challenge, then the design implications are clear: make getting to and configuring that feature simpler. While there is a wide range of accessibility features on common mobile platforms, only a small subset likely will be applicable for a given user, making it feasible for the system to suggest a few features to be controlled in an easy way. 
We predicted that older adults would not perceive themselves as needing accessibility features because of a lack of awareness or denial of ability changes. Yet, in the follow-up interviews, participants' reasons for not adopting accessibility features were either that they had forgotten how to use the features or that they lacked social support. Therefore, for older adults experiencing gradual ability changes in later life, factors such as attitudes towards learning technology, perceived ease-of-use, and social support might impact their adoption of accessibility features.

\section{Accessibility Features Do Not Accommodate Hesitance to Use Trial-and-Error}

Although the finding that many older adults do not use trialand-error to learn technology is not new [17], there may be more factors at play. The timing of the onset, type of onset (sudden or gradual), or the severity of an impairment may make older adults more or less receptive to using trial-anderror. Two of the four older adults who lived with a disability their entire lives reported using trial-and-error with technology as a result of their disability: P3 and P7 explained that because they depended on technology more than a person without a disability, they had to learn to use trial-anderror at an early age and were therefore more comfortable using this approach in their later years. However, for the majority of participants in our study who were experiencing gradual ability changes in later life, accessibility settings did not accommodate hesitance to use trial-and-error. In addition, participants and their family members noted that accessibility features were not accessible for individuals experiencing cognitive changes. Participants and family members observed that cognitive changes reduced older adults' ability to experiment with new features. Therefore, accessibility features and other mobile features that require configuration should be designed in a way that either supports exploration or reduces the negative consequences of making errors, for example, by allowing users to preview the changes of an accessibility setting across apps.

\section{Accessibility Features Do Not Easily Accommodate Combinations of Impairments}

Accessibility features do not accommodate older adults with combinations of impairments. None of the older adult participants in our study were experiencing an isolated ability change but were experiencing multiple ability changes simultaneously. Many of the features, such as dictation, Android TalkBack, Apple VoiceOver, zoom, or magnification require the participant to recall the sequence of steps to enable and control the feature, which is challenging for individuals experiencing cognitive changes. Other accessibility features require the participant to have proficient dexterity to be able to manipulate the feature (e.g., zoom and magnification require multiple taps and/or fingers).

\section{STUDY LIMITATIONS}

Our sample was self-selected by responding to recruiting advertisements for older adults experiencing ability changes; thus, we lacked older adults who are experiencing changes in their abilities but do not identify as such. Our sample also lacked educational diversity: all participants were highly educated, which might have impacted our findings (e.g., some older adults in our study might have tried the accessibility features demonstrated in the interview because they felt confident learning new skills). Furthermore, because our focus was on participants' subjective experiences, we did not measure their functional abilities; assessments of current abilities and changes in ability were all through self-report. Since we used these self-reported abilities to select accessibility features to introduce to participants, the inclusion of more objective measures may have resulted in the selection of different features. Finally, participants were asked to recall how changes in abilities affected their device use, whereas direct observation or contextual inquiry may have revealed additional insights that were not based on participants' ability to recall.

\section{CONCLUSION}

Although many older adults could benefit from mobile accessibility features, ours is the first empirical study exploring how older adults who are experiencing progressive ability changes adopt and use built-in mobile accessibility features. We found that accessibility features were considered hard to use, find, re-find, turn on and off, and configure, and so they were underutilized by most older adults. We also investigated attitudes toward ability changes and accessibility features, finding that older adults might not perceive themselves as needing accessibility features because of how these features are positioned relative to the concept of "disability." With these problems in mind, designers can improve mobile device and interface design to encourage older adults to discover accessibility features and use trial-and-error to explore their potential benefits. Accessibility features can be presented as mainstream enhancements to improve usability.

\section{ACKNOWLEDGEMENTS}

This research was funded under NSF grant IIS-1818594. Any opinions, findings, conclusions or recommendations expressed in our work are those of the authors and do not necessarily reflect those of any supporter.

\section{REFERENCES}

[1] Faranak Aminzadeh and Nancy Edwards. 1998. Exploring seniors' views on the use of assistive devices in fall prevention. Public Health Nursing 15 (4), 297304. DOI: 10.1111/j.1525-1446.1998.tb00353.x

[2] Monica Anderson and Andrew Perrin. 2017. Tech adoption climbs among older adults. http://www.pewinternet.org/2017/05/17/technologyuse-among-seniors/

[3] Lisa Anthony, YooJin Kim, and Leah Findlater. 2013. Analyzing user-generated youtube videos to understand touchscreen use by people with motor impairments. Proceedings of CHI 2013. New York: ACM Press, 1223-1232. DOI: 10.1145/2470654.2466158 
[4] Hugh Beyer and Karen Holtzblatt. 1999. Contextual design. Interactions 6, 32-42. ISBN: 9780080503042

[5] Aimée K. Bright and Lynne Coventry. 2013. Assistive technology for older adults. Proceedings of the 6th International Conference on Pervasive Technologies Related to Assistive Environments. New York: ACM Press, 1-4. DOI: 10.1145/2504335.2504344

[6] Nancy A. Brooks. 1991. Users' responses to assistive devices for physical disability. Social Science \& Medicine 32 (12), 1417-1424. https:/www.ncbi.nlm.nih.gov/pubmed/1831289

[7] Mario Conci, Fabio Pianesi, and Massimo Zancanaro. 2009. Useful, social and enjoyable: Mobile phone adoption by older people. In T. Gross, J. Gulliksen, P. Kotzé, L. Oestreicher, P. Palanque, R.O. Prates, \& M. Winckler (eds.), Human-Computer Interaction -INTERACT 2009. Berlin, Heidelberg: Springer Berlin Heidelberg. DOI: 10.1007/978-3-642-03655-2

[8] Fred D. Davis Jr. 1986. A technology acceptance model for empirically testing new end-user information systems: Theory and results. Massachusetts Institute of Technology. http://hdl.handle.net/1721.1/15192

[9] Leah Findlater, Jon E. Froehlich, Kays Fattal, Jacob O. Wobbrock, and Tanya Dastyar. 2013. Age-related differences in performance with touchscreens compared to traditional mouse input. Proceedings of CHI 2013. New York: ACM Press, 343-346. DOI: $10.1145 / 2470654.2470703$

[10] Rachel Franz, Leah Findlater, and Jacob O. Wobbrock. 2018. Lost in transition: The importance of conceptualizing aging as a process in accessibility research. In Workshop on "Designing Interactions for the Ageing Populations.” CHI 2018. Montreal, 53-58. https://faculty.washington.edu/wobbrock/pubs/chi18.03.pdf

[11] Ann E. Gerike. 1990. On gray hair and oppressed brains. Journal of Women \& Aging 2 (2), 35-46. DOI: 10.1300/J074v02n02_05

[12] Rachael Gooberman-Hill and Shah Ebrahim. 2007. Making decisions about simple interventions: Older people's use of walking aids. Age and Ageing 36 (5), 569-573. DOI: 10.1093/ageing/afm095

[13] Toshiyuki Hagiya, Toshiharu Horiuchi, and Tomonori Yazaki. 2016. Typing Tutor: Individualized tutoring in text entry for older adults based on input stumble detection. Proceedings of CHI 2016. New York: ACM Press, 733-744. DOI: 10.1145/2858036.2858455

[14] Shaun K. Kane, Chandrika Jayant, Jacob O. Wobbrock, and Richard E. Ladner. 2009. Freedom to roam: A study of mobile device adoption and accessibility for people with visual and motor disabilities. Proceedings of ASSETS 2009. New York: ACM Press, 115-122. DOI: $10.1145 / 1639642.1639663$
[15] Sri Kurniawan, Murni Mahmud, and Yanuar Nugroho. 2006. A study of the use of mobile phones by older persons. Extended Abstracts of CHI 2006. New York: ACM Press, 989-994. DOI: 10.1145/1125451.1125641

[16] Chaiwoo Lee and Joseph F. Coughlin. 2014. PERSPECTIVE: Older adults' adoption of technology: An integrated approach to identifying determinants and barriers. Journal of Product Innovation Management 32 (5), 747-759. DOI: 10.1111/jpim. 12176

[17] Rock Leung, Charlotte Tang, Shathel Haddad, Joanna Mcgrenere, Peter Graf, and Vilia Ingriany. 2012. How older adults learn to use mobile devices. $A C M$ Transactions on Accessible Computing 4 (3), 1-33. DOI: 10.1145/2399193.2399195

[18] Aqueasha Martin-Hammond, Abdullah Ali, Catherine Hornback, and Amy K. Hurst. 2015. Understanding design considerations for adaptive user interfaces for accessible pointing with older and younger adults. Proceedings of W4A 2015. New York: ACM Press, 110. DOI: $10.1145 / 2745555.2746645$

[19] Kathleen A. Martin, Mark R. Leary, and W. Jack Rejeski. 2000. Self-presentational concerns in older adults: Implications for health and well-being. Basic and Applied Social Psychology 22 (3), 169-179. DOI: 10.1207/S15324834BASP2203_5

[20] Ronald E. McGaughey, Steven M. Zeltmann, and Mark E. McMurtrey. 2013. Motivations and obstacles to smartphone use by the elderly: Developing a research framework. International Journal of Electronic Finance 7, (3/4), 177-195.

DOI: 10.1504/IJEF.2013.058601

[21] Lilian Genaro Motti, Nadine Vigouroux, and Philippe Gorce. 2013. Interaction techniques for older adults using touchscreen devices. Proceedings of IHM 2013 New York: ACM Press, 125-134.

DOI: $10.1145 / 2534903.2534920$

[22] Barbara Barbosa Neves, Rachel L. Franz, Cosmin Munteanu, Ronald Baecker, and Mags Ngo. 2015. "My hand doesn't listen to me!'”: Adoption and evaluation of a communication technology for the 'oldest old.' Proceedings of CHI 2015. New York: ACM Press, 1593-1602. DOI: 10.1145/2702123.2702430

[23] Alex Olwal, Dimitris Lachanas, and Ermioni Zacharouli. 2011. OldGen: Mobile phone personalization for older adults. Proceedings of $\mathrm{CHI}$ 2011. New York: ACM Press, 3393-3396. DOI: 10.1145/1978942.1979447

[24] Michael Quinn Patton. 2001. Qualitative Evaluation and Research Methods. SAGE Publications, Inc. ISBN: 0761919716

[25] Yi-Hao Peng, Muh-Tarng Lin, Yi Chen, TzuChuan Chen, Pin Sung Ku, Paul Taele, Chin Guan Lim, and Mike Y. Chen. 2019. PersonalTouch: Improving 
touchscreen usability by personalizing accessibility settings based on individual user's touchscreen interaction. Proceedings of CHI 2019. New York: ACM Press, 1-11. DOI: 10.1145/3290605.3300913

[26] Maribel Pino, Mélodie Boulay, François Jouen, and Anne-Sophie Rigaud. 2015. "Are we ready for robots that care for us?" Attitudes and opinions of older adults toward socially assistive robots. Frontiers in Aging Neuroscience 7 (141), 1-15.

DOI: 10.3389/fnagi.2015.00141

[27] Anne Marie Piper, Robin Brewer, and Raymundo Cornejo. 2017. Technology learning and use among older adults with late-life vision impairments. Universal Access in the Information Society 16 (3), 699-711. DOI: 10.1007/s10209-016-0500-1

[28] Anne Marie Piper, Ross Campbell, and James D. Hollan. 2010. Exploring the accessibility and appeal of surface computing for older adult health care support. Proceedings of CHI 2010. New York: ACM Press, 907-916. DOI: 10.1145/1753326.1753461

[29] J.J. Ray. 1988. Lie scales and the elderly. Personality and Individual Differences 9 (2), 417-418. DOI: 10.1016/0191-8869(88)90106-7

[30] Karen Renaud and Judy van Biljon. 2008. Predicting technology acceptance and adoption by the elderly. In Proceedings of the 2008 Annual Research Conference of the South African Institute of Computer Scientists and Information Technologists on IT Research in Developing Countries: Riding the Wave of Technology (SAICSIT '08). New York: ACM Press, 210-219. DOI: $10.1145 / 1456659.1456684$

[31] Kristen Shinohara and Jacob O. Wobbrock. 2011. In the shadow of misperception. Proceedings of $\mathrm{CHI}$ 2011. New York: ACM Press, 705-714. DOI: $10.1145 / 1978942.1979044$

[32] Marlee M. Spafford, Debbie Laliberte Rudman, Beverly D. Leipert, Lisa Klinger, and Suzanne Huot. 2010. When self-presentation trumps access: why older adults with low vision go without low-vision services. Journal of Applied Gerontology 29 (5), 579-602. DOI: $10.1177 / 0733464809345494$

[33] Robert Steele, Amanda Lo, Chris Secombe, and Yuk Kuen Wong. 2009. Elderly persons' perception and acceptance of using wireless sensor networks to assist healthcare. International Journal of Medical Informatics 78 (12), 788-801. DOI: 10.1016/j.ijmedinf.2009.08.001

[34] Christian Stößel, Hartmut Wandke, and Lucienne Blessing. 2010. Gestural interfaces for elderly users: Help or hindrance? In Stefan Kopp and Ipke Wachsmuth (eds.), Gesture in Embodied Communication and Human-Computer Interaction. Berlin, Heidelberg: Springer Berlin Heidelberg. 269280. DOI: $10.1007 / 978-3-642-12553-9 \_24$
[35] Sarit Felicia Anais Szpiro, Shafeka Hashash, Yuhang Zhao, and Shiri Azenkot. 2016. How people with low vision access computing devices. Proceedings of ASSETS 2016. New York: ACM Press, 171-180. DOI: $10.1145 / 2982142.2982168$

[36] Viswanath Venkatesh, Michael G. Morris, Gordon B. Davis, and Fred D. Davis. 2003. User acceptance of information technology: Toward a unified view. MIS Quarterly 27 (3), 425-478. DOI: 10.2307/30036540

[37] John Vines, Gary Pritchard, Peter Wright, Patrick Olivier, and Katie Brittain. 2015. An age-old problem: Examining the discourses of ageing in HCI and strategies for future research. ACM Transactions on Computer-Human Interaction 22 (1), 1-27. DOI: $10.1145 / 2696867$

[38] Chat Wacharamanotham, Jan Hurtmanns, Alexander Mertens, Martin Kronenbuerger, Christopher Schlick, and Jan Borchers. 2011. Evaluating Swabbing: A touchscreen input method for elderly users with tremor. Proceedings of CHI 2011. New York: ACM Press, 623-626. DOI: 10.1145/1978942.1979031

[39] Kieran Walsh and Aoife Callan. 2011. Perceptions, preferences, and acceptance of information and communication technologies in older-adult community care cettings in Ireland: A case-study and ranked-care program analysis. Ageing International 36 (1), 102122. DOI: $10.1007 / \mathrm{s} 12126-010-9075-\mathrm{y}$

[40] Jacob O. Wobbrock, Krzysztof Z. Gajos, Shaun K. Kane, and Gregg C. Vanderheiden. 2018. Ability-based design. Communications of the ACM 61 (6), 62-71. DOI: $10.1145 / 3148051$

[41] Jacob O. Wobbrock, Shaun K. Kane, Krzysztof Z. Gajos, Susumu Harada, and Jon Froehlich. 2011. Ability-based design: Concept, principles and examples. ACM Transactions on Accessible Computing 3 (3), 1-36. DOI: 10.1145/1952383.1952384

[42] 2017. World Population Ageing 2017. New York. http://www.un.org/en/development/desa/populati on/publications/pdf/ageing/WPA2017_Highlights.pdf 